\title{
Increasing resistance rate to carbapenem among blood culture isolates of Klebsiella pneumoniae, Acinetobacter baumannii and Pseudomonas aeruginosa in a university-affiliated hospital in China, 2004-2011
}

\author{
Xiaoli Zhang ${ }^{1,2}$, Bing $\mathrm{Gu}^{2}$, Yaning $\mathrm{Mei}^{2}$, Yi Wen ${ }^{2}$ and Wenying $\mathrm{Xia}^{2}$
}

The objective of this study is to investigate the profile of antimicrobial resistance of Gram-negative bacteria in blood cultures from 2004-2011. Pathogens from positive blood cultures were subcultured, and identified in the First Affiliated Hospital of Nanjing Medical University from January 2004 to December 2011. The antibiotic resistance pattern was analyzed by WHONET 5.4. A total of 1224 cases of Gram-negative bacterial isolates were documented, accounting for $38.6 \%$ of the total pathogens isolated from positive blood cultures in the 8-year period. The isolation rates of Klebsiella pneumoniae and Acinetobacter baumannii increased nearly three times over the same time span. Most Gram-negative bacteria isolates, except the isolates of Pseudomonas aeruginosa, showed a significantly increased resistance rate to cephalosporins (in particular third/fourth generation cephalosporins). Noteworthy, the antimicrobial resistance of $K$. pneumoniae, $A$. baumannii and $P$. aeruginosa isolates to carbapenem (imipenem and meropenem) was significantly increased and the resistant rate to carbapenem was $>80.0 \%$ in A. baumannii in 2011. The results from PCR detection for carbapenemases were as follows: $82.8 \%(24 / 29)$ isolates of $K$. pneumoniae carried the $k p c-2$ gene; only three metallo-beta-lactamase-positive $P$. aeruginosa isolates were detected; and $93.1 \%$ (67/72) A. baumannii isolates were blaOXA-23 positive. The antimicrobial resistance rate of Gram-negative bacteria isolated from blood cultures significantly increased from 2004 to 2011, with significant resistance to the third/fourth generation cephalosporins and carbapenem.

The Journal of Antibiotics (2015) 68, 115-120; doi:10.1038/ja.2014.119; published online 3 September 2014

\section{INTRODUCTION}

Bloodstream infection (BSI) is a systemic infectious disease. Severe BSI can cause septic shock, disseminated intravascular coagulation and multiple organ dysfunction syndrome. BSI accounts for 10.0-20.0 mortality $\%$ of all nosocomial infections and is the eighth leading cause of mortality $(15.0 \%)$ in the United States. ${ }^{1-3}$ Blood culture is the most direct method for the detection of BSI in patients.

The epidemiology of invasive pathogens has changed dramatically over the years ${ }^{4-6}$ with an increase in resistance to many antibiotic drugs ${ }^{4}$ resulting in a reduction in therapeutic options. ${ }^{5}$ The emergence of antibiotic resistance has become a global public health problem, especially for Gram-negative bacteria, as there is a lack of novel antibiotics directed against these organisms. For example, carbapenem, the agents of last resort against multidrug-resistant Enterobacteriaceae, are under threat with a growing incidence of panresistant isolates. ${ }^{7,8}$ A study has observed that inadequate therapy is the common reason for antimicrobial resistance. ${ }^{9}$ Information about the detection of pathogens and antibiotic resistance pattern are now seen as decisive for optimizing treatment. ${ }^{10}$ Thus, surveillance of BSI pathogens along with their antimicrobial resistance patterns is the key for prevention.

In this study, the profile and the antibiotic resistance patterns of Gram-negative bacteria, isolated from the blood culture in the First Affiliated Hospital of Nanjing Medical University from January 2004 to December 2011 were retrospectively reviewed to provide information on changes in antibiotic resistance patterns over time, and then it would provide evidence for clinical therapy.

\section{MATERIALS AND METHODS}

Patients

Our study was undertaken in a general teaching hospital with 2200 hospital beds. All the blood culture specimens were collected between 1 January 2004 and 31 December 2011 (single bottle aerobic culture for most specimens). At least $5 \mathrm{ml}$ of blood was inoculated into each culture bottle. The blood culture was collected from patients with symptoms or signs of bacteremia before antibiotic therapy and sent immediately to the microbiology laboratory for

${ }^{1} Y$ Y Xing People's Hospital, Yixing, Jiangsu, China and ${ }^{2}$ Department of Laboratory Medicine, the First Affiliated Hospital of Nanjing Medical University, Nanjing, China Correspondence: Dr B Gu, Department of Laboratory Medicine, the First Affiliated Hospital of Nanjing Medical University, Guangzhou Road No. 300, Nanjing 210029, China. E-mail: gb20031129@163.com

Received 12 July 2013; revised 1 June 2014; accepted 30 July 2014; published online 3 September 2014 
processing. If the same organism was repeatedly isolated from the same patient, only the first isolate was included in the analysis. Collection and culture of the blood specimens were performed according to standard operating procedures, following the Clinical and Laboratory Standards Institute guidelines. ${ }^{11}$

\section{Bacterial identification}

The Bactec Fluorescent series 9240 (Becton Dickinson, Franklin Lakes, NJ USA) instrument was used for blood culture analysis. When a positive alert was detected, the particular bottle was removed and Gram stain and subculture were on blood agar and chocolate agar plates, respectively. Specifically for anaerobic cultures, an additional anaerobic blood agar was performed. Bottles with no or negative alert were kept in the instrument for 7 days and subsequently subcultured to confirm the absence of bacterial organisms before being regarded as negative bottles. Gram-negative bacterial isolates were confirmed by API system (Mérieux, Lyon, France) and the VITEK-2 compact automatic identification system (Mérieux).

\section{Antimicrobial susceptibility testing}

The antimicrobial susceptibility testing was performed predominantly by Kirby-Bauer disk diffusion method, following the Clinical and Laboratory Standards Institute guidelines. ${ }^{11}$ Susceptibilities to the following antibiotics were tested: cephalosporins (cefazolin, cefuroxime, ceftazidime, cefotaxime and cefepime), monobactams (aztreonam), carbapenem (imipenem and meropenem), compound agents (amoxicillin/clavulanate, piperacillin/ tazobactam and cefoperazone/sulbactam), aminoglycosides (amikacin) and fluoroquinolones (ciprofloxacin and levofloxacin). Microbiologic data were extracted from the laboratory information system and converted into a standard format using WHONET 5.4 (WHO, Geneva, Switzerland), with duplicates eliminated according to the guidelines of the Clinical and Laboratory Standards Institute guidelines. The control strains included were as follows: Escherichia coli ATCC 25922, Klebsiella pneumonia ATCC 700603 and Pseudomonas aeruginosa ATCC 27853.

\section{PCR-based detection of genes encoding carbapenemases}

All of the 130 carbapenem-resistant isolates, which includes one E. coli isolate, $29 \mathrm{~K}$. pneumoniae isolates, $28 \mathrm{P}$. aeruginosa isolates and 72 Acinetobacter baumannii isolates, were subjected to PCR analysis to detect genes encoding carbapenemases. These genes include blaOXA-23, blaOXA-24, blaOXA-48, blaIMP, blaVIM, blaNDM and blaKPC. PCR was performed on a PTC-100type PCR instrument (MJ Research Inc., USA). DNA was extracted as previously described. ${ }^{12}$ The reaction conditions were as follows: predenaturation at $94^{\circ} \mathrm{C}$ for $4 \mathrm{~min}$, followed by 30 amplification cycles of $94^{\circ} \mathrm{C}$

\section{Table 1 Primers used in this study}

\begin{tabular}{|c|c|c|c|}
\hline Primer & Sequence $\left(5^{\prime}-3^{\prime}\right)$ & Length & Reference \\
\hline OXA-23 & $\begin{array}{l}\text { F:GATGTGTCATAGTATTCGTCG } \\
\text { R:TCACAACAACTAAAAGCACT }\end{array}$ & 1067 & 1 \\
\hline OXA-24 & $\begin{array}{l}\text { F:GTACTAATCAAAGTTGTGAA } \\
\text { R:TTCCCCTAACATGAATTTGT }\end{array}$ & 1024 & 1 \\
\hline OXA-48 & $\begin{array}{l}\text { F:TTGGTGGCATCGATTATCGG } \\
\text { R:GAGCACTTCTTTTGTGATGGC }\end{array}$ & 744 & 2 \\
\hline $\mathrm{KPC}$ & $\begin{array}{l}\text { F:CGTCTAGTTCTGCTGTCTTG } \\
\text { R:CTTGTCATCCTTGTTAGGCG }\end{array}$ & 798 & 3 \\
\hline NDM & $\begin{array}{l}\text { F:ATGGAATTGCCCAATATTATGC } \\
\text { R:CGAAAGTCAGGCTGTGTTG }\end{array}$ & 490 & 4 \\
\hline VIM & $\begin{array}{l}\text { F:GTTTGGTCGCATATCGCAAC } \\
\text { R:AATGCGCAGCACCAGGATAG }\end{array}$ & 382 & 5 \\
\hline IMP & $\begin{array}{l}\text { F:GAAGGCGTTTATGTTCATAC } \\
\text { R:GTATGTTTCAAGAGTGATGC }\end{array}$ & 587 & 5 \\
\hline
\end{tabular}

Abbreviations: IMP, Imipenemase; KPC, Klebsiella pneumoniae Carbapenemase; NDM, New Delhi metallo-beta-lactamase; OXA, oxacillinase; VIM, Verona imipenemase. for $1 \mathrm{~min}, 55^{\circ} \mathrm{C}$ for $1 \mathrm{~min}$ and $72^{\circ} \mathrm{C}$ for $1.5 \mathrm{~min}$, with a final extension step of $72^{\circ} \mathrm{C}$ for $10 \mathrm{~min}$. Primers used in this study were listed in Table $1^{13-17}$ Purification of PCR products and DNA sequencing were performed by GenScript Co., Ltd Similarity and alignment searches for the nucleotide sequences were analyzed using BLAST (http://www.ncbi.nlm.nih.gov/BL AST).

\section{Statistical analysis}

Statistical analysis was accomplished by WHONET 5.4, the microbiology laboratory data management software recommended by World Health Organization. If the number of isolates was $<10$, the antimicrobial susceptibility data were not included in the overall analysis.

\section{RESULTS}

\section{Pathogens distribution}

A total of 18745 blood culture samples were collected over a period of 8 years, with a pathogen-positive rate of $16.9 \%$ (3168/18745). Of those pathogens, $38.6 \%$ (1224/3168) were Gram-negative bacteria. An increased trend in the isolation rate of Gram-negative bacteria, from $32.2 \%(94 / 292)$ in 2004 to $47.5 \%$ (286/602) in 2011, was observed, greater than that of Gram-positive bacteria (45.0\%) in 2011. K. pneumoniae and A. baumannii were found increased observably in this study. The top nine frequently detected bacterial species or groups were: coagulase-negative staphylococci, E. coli, Staphylococcus aureus, K. pneumoniae, fungi, Streptococcus, Enterococcus, A. baumannii and P. aeruginosa (Figure 1).

\section{Antimicrobial resistance of Enterobacteriaceae}

An increased resistance rate to cephalosporins was seen among most Enterobacteriaceae species. In E. coli, the resistant rate was $>75.0 \%$ to all cephalosporins in 2011, and significantly increased trends were observed to cefuroxime, ceftazidime, cefotaxime and cefepime (Table 2). Resistance to cephalosporins was also seen for K. pneumoniae isolates during the study period. For example, the resistance rate to ceftazidime was $15.4 \%$ in 2005 , but increased significantly to $62.5 \%$ in 2011 . The same trend was also observed in terms of resistance to other cephalosporins (Table 3 ).

Resistance to antibiotics containing $\beta$-lactamase inhibitors such as amoxicillin/clavulanate, cefoperazone/sulbactam and piperacillin/ tazobactam also showed increasing trends in both E. coli and $K$. pneumonia isolates. Although E. coli isolates retained high susceptibility to carbapenem (imipenem and meropenem), $K$. pneumoniae isolates showed significantly increased resistance rates to imipenem and meropenem over the 8 -year period, from $0.0 \%$ in 2005 to almost $30.0 \%$ in 2011 (Tables 2 and 3). No obvious increasing trend was found for resistance to aztreonam, amikacin and levofloxacin in either E. coli or K. pneumonia isolates. The resistance rate to amikacin was relatively low compared to the three antibiotics mentioned above for both E. coli and K. pneumonia isolates.

\section{Antimicrobial resistance of non-fermenting bacteria}

Extremely high levels of cephalosporin resistance were observed for A. baumannii. In 2011, the resistance rates to ceftazidime, cefotaxime and cefepime were 95.6, 100.0 and 91.3\%, respectively. For $P$. aeruginosa isolates, the resistance rates to ceftazidime and cefepime were $<40.0 \%$, however, a resistance rate of $85.7 \%$ was observed to cefotaxime (Tables 4 and 5). Compared to A. baumannii isolates, $P$. aeruginosa isolates showed relatively lower resistance rate to compound agents $(<45.0 \%)$. Strikingly, both $P$. aeruginosa and A. baumannii isolates showed significant increase in carbapenem resistance during the study period. In 2008, the resistance rates to imipenem and meropenem for $P$. aeruginosa were 7.1 and $7.6 \%$, 


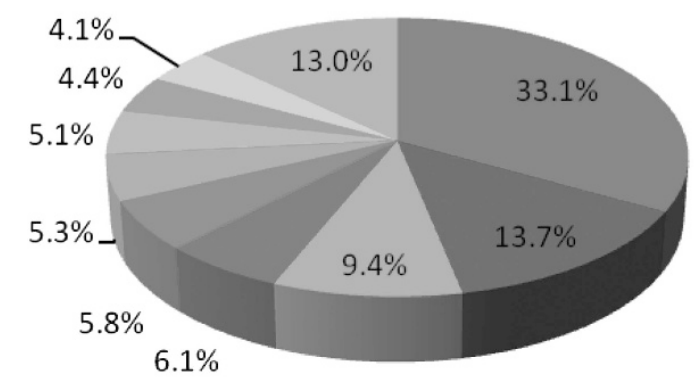

CONS

Escherichia coli

Staphylococcus aureus

Klebsiella pneumoniae

Fungi

- Streptococcus

- Enterococcus

- Acinetobacter baumannii

Pseudomonas aeruginosa

other bacteria

Figure 1 The distribution of top nine pathogens in blood cultures from 2004 to 2011 in the First Affiliated Hospital of Nanjing Medical University. CoNS is coagulase-negative staphylococci. A full color version of this figure is available at The Journal of Antibiotics journal online.

Table 2 Resistance of Escherichia coli, 2004 to 2011

\begin{tabular}{|c|c|c|c|c|c|c|c|c|}
\hline Antibiotics & $\begin{array}{c}2004 \\
(n=19) \\
(r=6.5 \%)\end{array}$ & $\begin{array}{c}2005 \\
(n=41) \\
(r=13.5 \%)\end{array}$ & $\begin{array}{c}2006 \\
(\mathrm{n}=46) \\
(\mathrm{r}=12.5 \%)\end{array}$ & $\begin{array}{c}2007 \\
(n=64) \\
(r=18.2 \%)\end{array}$ & $\begin{array}{c}2008 \\
(\mathrm{n}=60) \\
(\mathrm{r}=15.2 \%)\end{array}$ & $\begin{array}{c}2009 \\
(\mathrm{n}=71) \\
(\mathrm{r}=16.2 \%)\end{array}$ & $\begin{array}{c}2010 \\
(n=53) \\
(r=12.7 \%)\end{array}$ & $\begin{array}{c}2011 \\
(n=79) \\
(r=13.1 \%)\end{array}$ \\
\hline $\begin{array}{l}\text { Amoxicillin/ } \\
\text { clavulanate }\end{array}$ & 10.5 & 7.3 & 23.6 & 17.2 & 22.4 & 26.6 & 34.6 & 26.9 \\
\hline $\begin{array}{l}\text { Cefoperazone/ } \\
\text { sulbactam }\end{array}$ & 5.2 & 2.4 & 14.8 & 6.2 & 8.3 & 11.3 & 22.9 & 19.2 \\
\hline $\begin{array}{l}\text { Piperacillin/ } \\
\text { tazobactam }\end{array}$ & 0.0 & 4.9 & 13.5 & 18.3 & 11.9 & 10.3 & 16.7 & 10.1 \\
\hline Cefazolin & 66.7 & 68.4 & 79.2 & 62.9 & 70.7 & 76.4 & 64.6 & 75.9 \\
\hline Cefuroxime & 61.8 & 69.4 & 73.1 & 62.3 & 70.7 & 79.7 & 74.5 & 77.3 \\
\hline Ceftazidime & 31.6 & 16.8 & 16.4 & 22.2 & 71.2 & 78.8 & 66.7 & 75.9 \\
\hline Cefotaxime & 31.4 & 45.5 & 66 & 53.1 & 71.2 & 72.1 & 68.8 & 78.9 \\
\hline Cefepime & 47.4 & 26.8 & 37 & 41.9 & 70.2 & 77.9 & 70.6 & 75.9 \\
\hline Aztreonam & 57.9 & 23.7 & 27.8 & 31.1 & 70.7 & 75.4 & 70.6 & 75.9 \\
\hline Imipenem & 0.0 & 0.0 & 0.0 & 0.0 & 0.0 & 0.0 & 2.8 & 0.0 \\
\hline Meropenem & 0.0 & 0.0 & 0.0 & 0.0 & 0.0 & 0.0 & 2.8 & 0.0 \\
\hline Amikacin & 11.1 & 23.7 & 3.7 & 15.6 & 11.7 & 17.2 & 22.4 & 30.4 \\
\hline Levofloxacin & 72.2 & 57.5 & 66.7 & 79.7 & 71.4 & 82.8 & 55.3 & 60.3 \\
\hline
\end{tabular}

respectively; these rates increased rapidly to 45.9 and $46.5 \%$ in 2011 . In A. baumannii isolates, the resistance rates to imipenem and meropenem were both only $10.0 \%$ in 2008 , which raised to 83.5 and $82.1 \%$, respectively, in 2011 .

\section{Molecular characterization}

No genes encoding carbapenemases were found in E. coli isolates. Among the 29 isolates of K. pneumoniae, 82.8\% (24/29) carried the $k p c-2$ gene and no other carbapenemase-encoding gene was detected. Among the 28 carbapenem-resistant $P$. aeruginosa isolates, three metallo-beta-lactamase-positive strains were confirmed by PCR and DNA sequence analysis, including two strains positive for VIM-2 and one strain positive for IMP-1. Among the 72 isolates of A. baumannii, there were 67 isolates harboring an OXA-23-like gene and one strain harboring an OXA-24 gene. Neither blaOXA-48 nor blaNDM was amplified in any of these isolates.

\section{DISCUSSION}

In this study, E. coli remained the primary Gram-negative pathogen causing BSI in the recent 8-year period. In fact, Gram-negative bacteria have been encountered more frequently in blood culture than Gram-positive bacteria in studies conducted in Iran (52.4\% vs $47.6 \%)$ and in Northeastern Malaysia (55.2\% vs $42.1 \%) .{ }^{18,19}$ Because of the long-term use of antibiotics ${ }^{13,14}$ the incidence of antibiotic-resistant pathogens, such as A. baumannii and K. pneumoniae, have gradually increased. A substantial increase in the isolation rate of $K$. pneumoniae and A. baumannii was found during this study. The isolation rate of A. baumannii significantly increased from $1.8 \%$ (2005) to $7.6 \%$ (2011), more than that of $P$. aeruginosa since 2009. A. baumannii generally infects patients with low immunity, old age or immunosuppressive medications. In this study, most blood samples positive for A. baumannii came from departments of respiratory medicine, hematology, neurology, burn and intensive care units, because the patients in these sections were all in critical condition, 
Table 3 Resistance of Klebsiella pneumoniae, 2005-2011

\begin{tabular}{|c|c|c|c|c|c|c|c|}
\hline Antibiotics & $\begin{array}{c}2005 \\
(n=12) \\
(r=3.7 \%)\end{array}$ & $\begin{array}{c}2006 \\
(n=19) \\
(r=5.6 \%)\end{array}$ & $\begin{array}{c}2007 \\
(n=13) \\
(r=3.7 \%)\end{array}$ & $\begin{array}{c}2008 \\
(n=14) \\
(r=3.5 \%)\end{array}$ & $\begin{array}{c}2009 \\
(n=22) \\
(r=5.0 \%)\end{array}$ & $\begin{array}{c}2010 \\
(n=44) \\
(r=10.5 \%)\end{array}$ & $\begin{array}{c}2011 \\
(n=65) \\
(r=10.8 \%)\end{array}$ \\
\hline Amoxicillin/clavulanate & 16.7 & 5.6 & 25 & 21.4 & 15.8 & 55.6 & 45.2 \\
\hline $\begin{array}{l}\text { Cefoperazone/ } \\
\text { sulbactam }\end{array}$ & 7.7 & 5.9 & 15.4 & 7.1 & 7.1 & 37.1 & 37.7 \\
\hline Piperacillin/tazobactam & 15.4 & 5.9 & 23.1 & 7.7 & 19 & 36.8 & 50.8 \\
\hline Cefazolin & 50.0 & 52.2 & 62.5 & 28.6 & 28.6 & 71.1 & 64.5 \\
\hline Cefuroxime & 55.6 & 54.5 & 62.5 & 35.7 & 38.1 & 71.4 & 64.5 \\
\hline Ceftazidime & 15.4 & 13 & 12.5 & 28.6 & 23.8 & 73.7 & 62.5 \\
\hline Cefotaxime & 41.7 & 45.5 & 37.5 & 28.6 & 23.5 & 70.6 & 61.3 \\
\hline Cefepime & 23.1 & 18.2 & 20.0 & 28.6 & 23.8 & 73.7 & 66.7 \\
\hline Aztreonam & 33.3 & 21.7 & 20.0 & 30.8 & 23.8 & 73.7 & 64.1 \\
\hline Imipenem & 0.0 & 0.0 & 0.0 & 0.0 & 4.8 & 10.8 & 30.0 \\
\hline Meropenem & 0.0 & 0.0 & 0.0 & 0.0 & 4.8 & 8.7 & 35.5 \\
\hline Amikacin & 7.7 & 11.1 & 30.8 & 7.1 & 30.0 & 26.3 & 23.4 \\
\hline Levofloxacin & 15.4 & 23.5 & 23.1 & 20 & 13.3 & 42.1 & 47.6 \\
\hline
\end{tabular}

Table 4 Resistance of Pseudomonas aeruginosa, 2008-2011

\begin{tabular}{lcccc}
\hline & $\begin{array}{c}2008 \\
(\mathrm{n}=17) \\
(\mathrm{r}=4.3)\end{array}$ & $\begin{array}{c}2009 \\
(\mathrm{n}=17) \\
(\mathrm{r}=3.9)\end{array}$ & $\begin{array}{c}2010 \\
(\mathrm{n}=13) \\
(\mathrm{r}=2.9)\end{array}$ & $\begin{array}{c}2011 \\
(\mathrm{n}=37) \\
(\mathrm{r}=6.2)\end{array}$ \\
\hline $\begin{array}{l}\text { Antibiotics } \\
\text { sulbactam }\end{array}$ & 0.0 & 11.1 & 44.4 & 33.3 \\
Piperacillin/ & 12.5 & 29.4 & 44.4 & 32.4 \\
tazobactam & & & & \\
Ceftazidime & 5.9 & 5.9 & 33.3 & 37.8 \\
Cefotaxime & 82.4 & 100 & 77.8 & 85.7 \\
Cefepime & 17.6 & 17.6 & 33.3 & 27.8 \\
Aztreonam & 5.9 & 12.5 & 55.6 & 21.6 \\
Imipenem & 7.1 & 17.6 & 44.4 & 45.9 \\
Meropenem & 7.6 & 18.8 & 45.6 & 46.5 \\
Amikacin & 5.9 & 18.2 & 20 & 45.9 \\
Ciprofloxacin & 0.0 & 0.0 & 57.1 & 44.8 \\
Levofloxacin & 0.0 & 7.1 & 71.4 & 43.2 \\
\hline
\end{tabular}

' $r$ ' means the percentage of $P$. aeruginosa strains among all strains isolated from blood culture. In 2004-2007, the percentage were $7.2 \%(21 / 292), 4.0 \%(13 / 327), 2.9 \%(10 / 342)$ and $0.9 \%$ (3/352), respectively.

showing tracheostomy, low immunity, long-term hospitalization or suffering from various underlying diseases.

The resistance rate of Enterobacteriaceae to cephalosporins increased significantly, especially to third/fourth generation cephalosporins. The resistance rates of $E$. coli and $K$. pneumoniae to cephalosporins were all $>60.0 \%$ in 2011. Similar results were also reported in India, ${ }^{20}$ which was different from that in Europe and America. ${ }^{21,22}$ Our results also indicated that Enterobacteriaceae retained a high susceptibility to carbapenem, compared with that of non-fermenting bacteria. Nevertheless, $30.0 \%$ of $K$. pneumoniae isolates were imipenem-resistant in 2011 compared with $0.0 \%$ in 2004 , and the number was only $4.3 \%$ in the USA. ${ }^{23}$ This discrepancy is possibly related to the prevalence of Klebsiella pneumoniae Carbapenemase (KPC) carbapenemase. ${ }^{24}$ The results from PCR analysis indicate that the blaKPC-2 gene is the main reason for carbapenem resistance in $K$. pneumoniae isolates in our hospital.
Table 5 Resistance of Acinetobacter baumannii, 2008-2011

\begin{tabular}{lcccc}
\hline & $\begin{array}{c}2008 \\
(\mathrm{n}=11) \\
(\mathrm{r}=2.8 \%)\end{array}$ & $\begin{array}{c}2009 \\
(\mathrm{n}=26) \\
(\mathrm{r}=5.9 \%)\end{array}$ & $\begin{array}{c}2010 \\
(\mathrm{n}=28) \\
(\mathrm{r}=6.7 \%)\end{array}$ & $\begin{array}{c}2011 \\
(\mathrm{n}=46) \\
(\mathrm{r}=7.6 \%)\end{array}$ \\
\hline $\begin{array}{l}\text { Antibiotics } \\
\text { Amoxicillin/ }\end{array}$ & 81.8 & 83.3 & 90.5 & 94.7 \\
$\begin{array}{l}\text { Cefoperazone/ } \\
\text { sulbactam }\end{array}$ & 9.1 & 26.7 & 71.4 & 76.3 \\
Piperacillin/ & 54.5 & 80.8 & 85.7 & 95.7 \\
tazobactam & & & & \\
Ceftazidime & 63.6 & 84.0 & 95.0 & 95.6 \\
Cefotaxime & 72.7 & 96.0 & 100.0 & 100.0 \\
Cefepime & 54.5 & 76.0 & 76.2 & 91.3 \\
Aztreonam & 40.0 & 96.2 & 81.0 & 100 \\
Imipenem & 10.0 & 47.6 & 76.2 & 83.5 \\
Meropenem & 10.0 & 44.0 & 76.2 & 82.1 \\
Amikacin & 72.7 & 53.8 & 76.2 & 56.5 \\
Levofloxacin & 45.5 & 69.2 & 76.2 & 69.6 \\
\hline
\end{tabular}

' $r$ ' means the percentage of $A$. baumannii strains among all strains isolated from blood culture. In 2004-2007, the percentage were 3.1\% (9/292), 1.8\% (6/327), 2.0\% (7/342) and $2.6 \%$ (9/352), respectively.

P. aeruginosa is a common Gram-negative nosocomial pathogen. Because of the wide use of broad-spectrum antibiotics, the emergence of multidrug-resistant $P$. aeruginosa has continually increased, and the stains were often carbapenem-resistant, which can severely limit therapeutic options. ${ }^{25}$ In this study, only the resistance rate of $P$. aeruginosa to carbapenem showed an increasing trend from $0.0 \%$ in 2004 to $>30.0 \%$ in 2011. Moreover, carbapenem-resistant $P$. aeruginosa has also been observed more frequently in Europe and North America. ${ }^{26,27} \mathrm{Li}$ et al. ${ }^{28}$ found that the efflux pump on the cell membrane of $P$. aeruginosa is one of the main reasons for its multidrug resistance and the outer membrane protein OprM is the most common one among six efflux pumps. A study from Korea demonstrated that the major factor leading to carbapenem resistance in $P$. aeruginosa isolates is a result of a loss or decreased levels of OprD that leads to cell membrane impermeability. ${ }^{29}$ Moreover, only three $P$. aeruginosa isolates were shown to carry the genes coding for 
carbapenemases, which indicated that carbapenemases were not the main mechanism for carbapenem resistance in $P$. aeruginosa in this study.

Noteworthy, the antibiotic resistance of A. baumannii has become increasingly serious especially in terms of resistance to carbapenem (imipenem and meropenem), with the resistance rate rising from $10.0 \%$ in 2008 to $>80.0 \%$ in 2011 . Similar resistance rates were also found in Londrina, Brazil. ${ }^{30}$ Earlier reports indicated that A. baumannii was the cause of infection outbreaks in hospitals, ${ }^{31,32}$ and the multidrug resistance patterns among A. baumannii isolates often left carbapenem as the only effective treatment for severe infections. ${ }^{32}$ Today, carbapenem-resistant A. baumannii stains are an emerging threat worldwide and have been observed in several countries, ${ }^{30,31,33-37}$ although rare in Canada. ${ }^{38}$ Once carbapenemresistant $A$. baumannii emerged, a pan-drug resistant $A$. baumannii appeared; consequently, an infected patient has little chance of an effective therapy. ${ }^{39}$ As carbapenemases are probably the major contributor to carbapenem resistance, ${ }^{40}$ the resistance of A. baumannii to carbapenem is most concerning. ${ }^{41}$ The major mechanism of carbapenem resistance in A. baumannii is the production of OXA $\beta$-lactamases, primarily OXA-23, OXA-66 and OXA-58. ${ }^{24}$ In our study, most carbapenem-resistant A. baumannii isolates were positive for blaOXA-23, which indicated that the presence of blaOXA-23 gene was the main factor associated with carbapenem resistance in A. baumannii.

In recent years, the emergence of blaNDM was reported in some provinces of mainland China, ${ }^{42-44}$ with an outbreak of NDM-1-producing K. pneumonia having been reported. ${ }^{45}$ In our study, we did not find any NDM-positive isolate. The carbapenem resistance is showing an increasing trend worldwide that could be due to the following factors. ${ }^{46}$ First, the transmission mode of genes encoding carbapenemases accelerates the spread of carbapenem resistance. ${ }^{47}$ Most genes are located on mobile gene structures and can transmit on a high speed among various bacterial species. Second, the overprescribing of carbapenem is an important factor leading to carbapenem resistance. The prevalence of extended-spectrum $\beta$-lactamase (ESBL)-producing bacteria makes carbapenem a conventional choice in the treatment of multidrug-resistant bacteria, and the long-term use of carbapenem is the main factor causing carbapenem-resistant bacterial infection in some inpatients. Other factors such as international travel, ${ }^{48}$ inefficient measures to control hospital infection and frequent use of invasive medical devices may also be involved in the spread of carbapenem-resistant bacteria.

Our study indicated that Gram-negative bacteria have become a more significant cause of BSI, and that antimicrobial resistance is severe in this hospital. This is likely due to several factors with the major factor appearing to be excessive use of antibiotics. ${ }^{49}$ The total annual consumption of carbapenem markedly increased, 192 times more in 2009 than in 2001 in our hospital..$^{50}$ An association between the amount of carbapenem consumption and the resistance rate to carbapenem have been reported..$^{20,51,52}$ Now a related policy has been formulated in our hospital, which restricted the right of prescribing certain antibiotics including carbapenems to specified senior physicians.

In conclusion, this 8-year study highlights the increase in the isolation rate of Gram-negative bacteria causing BSI and confirms the increase in resistance to certain antibiotics. Reducing antibiotic consumption by proper prescription should be the primary step to minimizing resistance, but antibiotic surveillance is needed in order to enhance the effectiveness of medication.

\section{CONFLICT OF INTEREST}

The authors declare no conflict of interest.

\section{ACKNOWLEDGEMENTS}

This research was funded by the National Natural Science Foundation of China (No. 81000754) and received a grant from the Key Laboratory for Laboratory Medicine of Jiangsu Province of China (No. XK201114).

1 Wenzel, R. P. \& Edmond, M. B. The impact of hospital-acquired bloodstream infections. Emerg. Infect. Dis. 7, 174-177 (2001).

2 Martin, G. S., Mannino, D. M., Eaton, S. \& Moss, M. The epidemiology of sepsis in the United States from 1979 through 2000. N. Engl. J. Med. 348, 1546-1554 (2003).

3 Weinstein, M. P. et al. The clinical significance of positive blood cultures in the 1990s: a prospective comprehensive evaluation of the microbiology, epidemiology, and outcome of bacteremia and fungemia in adults. Clin. Infect. Dis. 24, 584-602 (1997).

4 Karchmer, A. W. Nosocomial bloodstream infections: organisms, risk factors, and implications. Clin. Infect. Dis. 31, 139-143 (2000).

5 Trick, W. E., Fridkin, S. K., Edwards, J. R., Hajjeh, R. A. \& Gaynes, R. P. Secular trend of hospital-acquired candidemia among intensive care unit patients in the United States during 1989-1999. Clin. Infect. Dis. 35, 627-630 (2002).

6 Hsueh, P. R. et al. Pandrug-resistant Acinetobacter baumannii causing nosocomial infections in a university hospital, Taiwan. Emerg. Infect. Dis. 8, 827-832 (2002).

7 Overturf, G. D. Carbapenemases: a brief review for pediatric infectious disease specialists. Pediatr. Infect. Dis. 29, 68-70 (2010).

8 Aubron, C., Poirel, L., Ash, R.J. \& Nordmann, P. Carbapenemase-producing Enterobacteriaceae, U.S. rivers. Emerg. Infect. Dis. 11, 260-264 (2005).

9 Reynolds, R. et al. Antimicrobial susceptibility of the pathogens of bacteraemia in the UK and Ireland 2001-2002: the BSAC Bacteraemia Resistance Surveillance Programme. J. Antimicrob. Chemother. 53, 1018-1032 (2004).

10 Chang, M. R. et al. Surveillance of pediatric infections in a teaching hospital in Mato Grosso do Sul, Brazil. Braz. J. Infect. Dis. 7, 149-160 (2003).

11 Clinical and Laboratory Standards Institute. Analysis and Presentation of Cumulative Susceptibility Test Data; Approved Guideline, 2nd ed. Document M39-A2 (vol. 25, no. 28). (Clinical and Laboratory Standards Institute, Wayne (PA), 2006).

$12 \mathrm{Gu}, \mathrm{B}$. et al. Prevalence and characterization of class I integrons among Pseudomonas aeruginosa and Acinetobacter baumannii isolates from patients in Nanjing, China. J. Clin. Microbiol. 45, 241-243 (2007).

13 Afzal-Shah, M., Woodford, N. \& Livermore, D. M. Characterization of OXA-25, OXA-26, and OXA-27, molecular class D $\beta$-lactamases associated with carbapenem resistance in clinical isolates of Acinetobacter baumannii. Antimicrob. Agents Chemother. 45, 583-588 (2001).

14 Poirel, L., Héritier, C., Tolün, V. \& Nordmann, P. Emergence of oxacillinase-mediated resistance to imipenem in Klebsiella pneumoniae. Antimicrob. Agents Chemother. 48, 15-22 (2004)

15 Poirel, L., Walsh, T. R., Cuvillier, V. \& Nordmann, P. Multiplex PCR for detection of acquired carbapenemase genes. Diagn. Microbiol. Infect. Dis. 70, 119-123 (2011).

16 Revathi, G., Siu, L. K., Lu, P. L. \& Huang, L. Y. First report of NDM-1-producing Acinetobacter baumannii in East Africa. Int. J. Infect. Dis. 17, e1255-e1258 (2013).

17 Fallah, F., Borhan, R. S. \& Hashemi, A. Detection of bla (IMP) and bla (VIM) metallo- $\beta$ lactamases genes among Pseudomonas aeruginosa strains. Int. J. Burns Trauma 3, 122 (2013)

18 Pourakbari, B et al. Five-year evaluation of the antimicrobial susceptibility patterns of bacteria causing bloodstream infections in Iran. J. Infect. Dev. Ctries. 6, 120-125 (2012).

19 Hashairi, F., Hasan, H., Azlan, K. \& Deris, Z. Z. An eight-year review of blood culture and susceptibility among sepsis cases in an emergency department in Northeastern Malaysia. Trop. Biomed. 28, 599-605 (2011).

20 Datta, S et al. A ten year analysis of multi-drug resistant blood stream infections caused by Escherichia coli \& Klebsiella pneumonia in a tertiary care hospital. Indian J. Med. Res. 135, 907-912 (2012).

21 Glupczynski, Y., Delmee, M., Goossens, H. \& Struelens, M. Belgian Multicenter ICU Study Group. Distribution and prevalence of antimicrobial resistance among gramnegative isolates in intensive care units (ICU) in Belgian hospitals between 1996 and 1999. Acta Clin. Belg. 56, 297-306 (2001).

22 Barlow, M. \& Hall, B. G. Experimental prediction of the natural evolution of antibiotic resistance. Genetics 163, 1237-1241 (2003).

23 Sanchez, G. V. et al. Klebsiella pneumoniae antimicrobial drug resistance, United States, 1998-2010. Emerg. Infect. Dis. 19, 133-136 (2013).

24 Xiao, Y. H. et al. Epidemiology and characteristics of antimicrobial resistance in China. Drug. Resist. Updat. 14, 236-250 (2011).

25 Scheffer, M. C. et al. Intrahospital spread of carbapenem-resistant Pseudomonas aeruginosa in a University Hospital in Florianopolis, Santa Catarina, Brazil. Rev. Soc. Bras. Med. Trop. 43, 367-371 (2010)

26 Gales, A. C., Jones, R. N., Turnidge, J., Rennie, R. \& Ramphal, R. Characterization of Pseudomonas aeruginosa isolates: occurrence rates, antimicrobial susceptibility patterns and molecular typing in the global SENTRY antimicrobial surveillance program, 1997-1999. Clin. Infect. Dis. 32(suppl 2), S146-S155 (2001b). 
27 Jones, M. E. et al. Emerging resistance among bacterial pathogens in the intensive care unit-a European and North American Surveillance study (2000-2002). Ann. Clin. Microbiol. Antimicrob. 3, 14 (2004).

$28 \mathrm{Li}$, X. Z., Poole, K. \& Nikaido, H. Contributions of MexAB-OprM and an EmrE homolog to intrinsic resistance of Pseudomonas aeruginosa to aminoglycosides and dyes. Antimicrob. Agents Chemother. 47, 27-33 (2003).

29 Pai, H. et al. Carbapenem resistance mechanisms in Pseudomonas aeruginosa clinical isolates. Antimicrob. Agents Chemother. 45, 480-484 (2001).

30 Viana, G. F. et al. Evolution of antimicrobial resistance of Acinetobacter baumannii in a university hospital. Lett. Appl. Microbiol. 53, 374-378 (2011).

31 Morgan, D. J. et al. Multidrug-resistant Acinetobatcter baumannii in New York City-10 years into the epidemic. Infect. Control Hosp. Epidemiol. 30, 196-197 (2009).

32 Pournaras, S. et al. Outbreak of multiple clones of imipenem-resistant Acinetobacter baumannii isolates expressing OXA-58 carbapenemase in an intensive care unit. J. Antimicrob. Chemother. 57, 557-561 (2006).

33 Livermore, D. M. et al. Antimicrobial treatment and clinical outcome for infections with carbapenem and multiply-resistant Acinetobacter baumannii around London. Int. J. Antimicrob. Agents 35, 19-24 (2010).

34 Peleg, A. Y., Seifert, H. \& Paterson, D. L. Acinetobacter baumannii: emergence of a successful pathogen. Clin. Microbiol. Rev. 21, 538-582 (2008).

35 Tankovic, J et al. Characterization of a hospital outbreak of imipenem resistance Acinetobacter baumannii by phenotypic and genotypic typing methods. J. Clin. Microbiol. 32, 2677-2681 (1994).

36 Ying, C. M., Ling, T. K., Lee, C. C. \& Ling, J. M. Characterization of carbapenemresistant Acinetobacter baumannii in Shanghai and Hong Kong. J. Med. Microbiol. 55 799-802 (2006).

37 Park, Y. K. et al. Changes in antimicrobial susceptibility and major clones of Acinetobacter calcoaceticus-baumannii complex isolates from a single hospital in Korea over 7 years. J. Med. Microbiol. 61, 71-79 (2012).

$38 \mathrm{McCracken}, \mathrm{M}$ et al. Characterization of Acinetobacter baumannii and meropenemresistant Pseudomonas aeruginosa in Canada: results of the CANWARD2007-2009 study. Diagn. Microbiol. Infect. Dis. 69, 335-341 (2011).

39 Yoon, J., Urban, C., Terzian, C., Mariano, N. \& Rahal, J. J. In vitro double and triple synergistic activities of polymyxin $B$, imipenem, and rifampin against multidurg resistant Acinetobacter baumannii. Antimicrob. Agents Chemother. 48, 753-757 (2004).
40 Hawkey, P. M. \& Jones, A. M. The changing epidemiology of resistance. J. Antimicrob. Chemother. 64, 3-10 (2009).

41 Kempf, M. \& Rolain, J. M. Emergence of resistance to carbapenems in Acinetobacter baumannii in Europe: clinical impact and therapeutic options. Int. J. Antimicrob. Agents 39, 105-114 (2012)

42 Liu, Z. et al. Identification and characterization of the first Escherichia coli strain carrying NDM-1 gene in China. PLOS ONE 8, e66666 (2013).

$43 \mathrm{Hu}$, L. et al. Emergence ofbla NDM-1 among Klebsiella pneumonia ST15 and nove ST1031 clinical isolates in China. Diagn. Microbiol. Infect. Dis. 75, 373-376 (2013).

44 Yang, J. et al. Dissemination and characterization of NDM-1-producing Acinetobacter pittii in an intensive care unit in China. Clin. Microbiol. Infect. 18, 506-513 (2012).

45 Wang, X. et al. An outbreak of a nosocomial NDM-1-producing Klebsiella pneumoniae ST147 at a Teaching Hospital in Mainland China. Microb. Drug Resist. 20, 144-149 (2014).

46 Sievert, D. M. et al. Antimicrobial-resistant pathogens associated with healthcare associated infections: summary of data reported to the National Healthcare Safety Network at the Centers for Disease Control and Prevention, 2009-2010. Infect. Control Hosp. Epidemiol. 34, 1-14 (2013).

47 Naas, T. et al. Genetic structures at the origin of acquisition of the beta-lactamase bla KPC gene. Antimicrob. Agents Chemother. 52, 1257-1263 (2008).

48 Yong, D. et al. Characterization of a new metallo-beta-lactamase gene, bla(NDM-1), and a novel erythromycin esterase gene carried on a unique genetic structure in Klebsiella pneumoniae sequence type 14 from India. Antimicrob. Agents Chemother. 53, 5046-5054 (2009).

49 Kotwani, A., Holloway, K. \& Chaudhury, R. R. Methodology for surveillance of antimicrobials use among out-patients in Delhi. Indian J. Med. Res. 129, 555-560 (2009).

$50 \mathrm{Xia}, \mathrm{W}$. Y. et al. Changing trend of antimicrobial resistance among pathogens isolated from lower respiratory tract at a university-affiliated hospital of China, 2006-2010. J. Thorac. Dis. 4, 284-291 (2012).

51 Livermore, D. M., Hope, R., Brick, G., Lillie, M. \& Reynolds, R; BSAC Working Parties on Resistance Surveillance. Non-susceptibility trends among Enterobacteriaceae from bacteraemias in the UK and Ireland, 2001-2006. J. Antimicrob. Chemother. 62(supp 2), ii41-ii54 (2008).

$52 \mathrm{Cao}$, J. et al. Correlation between carbapenem consumption and antimicrobial resistance rates of Acinetobacter baumannii in a university-affiliated hospital in China. J. Clin. Pharmacol. 53, 96-102 (2013). 